\title{
Avaliação da Aceitação de Tarefas Crowdsourcing: Uma Experiência do Usuário no Mundo Real
}

\author{
Maria Clara Pestana, Vaninha Vieira \\ ${ }^{1}$ Instituto de Matemática e Estatística - Universidade Federal da Bahia - Salvador - BA \\ - Brasil \\ clarapestana91@gmail.com, vaninha@ufba.br
}

\begin{abstract}
Crowdsourcing initiatives consider people intelligence for performing tasks to solve hard problems for machines. The goal of this research is to investigate the acceptance of users that participate of this type of initiatives in mobile devices. The acceptance of tasks by users will depend of the allocated task, considering the contextual aspect in which the user is involved in the moment that receives the task. The experiment will be conducted close to the real world, evaluating the users under real problems and using established crowdsourcing applications.
\end{abstract}

Resumo. Iniciativas crowdsourcing consideram a inteligência das pessoas para realizar tarefas resolvendo problemas dificeis para máquinas. O objetivo desta pesquisa é investigar a aceitação dos usuários participantes de iniciativas crowdsourcing presentes em dispositivos móveis. A aceitação das tarefas por esses usuários vai depender da tarefa que lhes são alocadas, considerando aspectos do contexto em que o mesmo se encontra no momento em que recebe a tarefa. Será realizado um experimento que se aproxime do mundo real, avaliando usuários com problemas reais e utilizando aplicações crowdsourcing existentes.

\section{Introdução}

A ideia de utilizar o poder da multidão, conhecido por Crowdsourcing (Howe, 2016), vem surgindo cada vez mais nos mais diversos tipos de iniciativas que utilizam da colaboração para resolver problemas levantados. Segundo Brabham (2013), iniciativas crowdsourcing são gerenciadas por uma organização ou indivíduos que enviam tarefas.

$\mathrm{Na}$ administração pública, por exemplo, o governo pode encontrar soluções para gerenciar as cidades consultando os cidadãos. Algumas aplicações populares podem ser consideradas crowdsourcing, como: a plataforma Amazon MTurk ${ }^{1}$ para cadastro de tarefas de inteligência humana em troca de valor monetário; o Pinterest ${ }^{2}$ disponibiliza

\footnotetext{
${ }^{1}$ www.mturk.com

${ }^{2}$ www.pinterest.com
} 
um banco para cadastro de ideias; a plataforma DesignCrowd ${ }^{3}$ permite que organizações que precisam de um designer publiquem seus projetos para designers cadastrados.

Através de observações cotidianas, podemos notar implicações no funcionamento dos sistemas crowdsourcing que podem afetar experiência no uso do dispositivo móvel. Por exemplo, uma alta requisição de tarefas ocasiona sobrecarga de notificações no aparelho, que podem resultar em problemas de motivação e engajamento dos usuários nas tarefas propostas. Porém, esses problemas podem ser minimizados se aplicada uma distribuição apropriada dessas tarefas visando os potenciais usuários do sistema que irão resolve-la. Essa distribuição pode prover melhores resultados tanto para o usuário que está solicitando, tanto para a aceitação do usuário que resolverá a tarefa.

A presente pesquisa pretende investigar a aceitação dos usuários participantes de iniciativas crowdsourcing presentes em dispositivos móveis. Nessa pesquisa, considerase o termo "contribuinte" para os usuários que colaboram com as tarefas do sistema, e os idealizadores das tarefas, pelo termo "solicitantes". A aceitação desses usuários vai depender da tarefa que lhes são alocadas, considerando aspectos do contexto em que o mesmo se encontra no momento em que recebe a tarefa. Os aspectos de contexto aqui trabalhados são baseados no modelo de distribuição de tarefas crowdsourcing ConTask Model (Pestana e Vieira, 2018) que apresenta parâmetros que podem ser utilizados como critérios para distribuir tais tarefas, preferencialmente em dispositivos móveis.

No experimento prévio realizado com o Contask Model, foi constatado que a variação dos elementos de contexto definidos no modelo pode afetar a aceitação dos trabalhadores das seguintes maneiras: (i) tarefas localizadas junto aos trabalhadores podem ser mais aceitáveis; (ii) a taxa de rejeição das tarefas pode ser reduzida por um relacionamento mais restrito entre as entidades do sistema; (ii) a alta frequência no local pode ser crucial para aceitar a tarefa; (iii) as atividades dos trabalhadores podem influenciar na aceitação (ex. Em espera); (iv) tarefas que demandam um alto esforço para serem concluídas podem ser mais facilmente rejeitadas; (v) o conhecimento prévio do assunto da tarefa pelo trabalhador pode ser crucial para a aceitação da tarefa.

Visto que esse experimento abrangeu uma quantidade pequena de pessoas e que a proposta crowdsourcing é que haja multidões, faz-se necessário um estudo de avaliação que colete a aceitação advindas de um grande contingente de pessoas por meio de plataformas já estabelecidas. Outro aspecto que poderia ser aprimorado é quanto à necessidade de coletar aspectos sobre a experiência do usuário (UX) (Roto et al, 2009). Esse aspecto promete coletar informações de como os usuários se sentem ao utilizar determinados produtos.

A maioria dos experimentos em pesquisas publicadas na área de crowdsourcing são em sua maioria baseadas em simulações. No caso da presente pesquisa, pretende-se fazer uma investigação que se aproxime do mundo real, por meio de aplicações crowdsourcing existentes. A avaliação visa investigar os parâmetros desses sistemas que podem ser decisivos na aceitação da tarefa pelos contribuintes. Esses parâmetros são baseados nos aspectos tratados nos modelos discutidos na seção 2 da fundamentação teórica, sendo estes o modelo ConTask e o modelo Guo et al. (2018).

\footnotetext{
${ }^{3}$ www.designcrowd.com
} 


\section{Fundamentação Teórica}

A presente pesquisa é uma continuação de uma investigação prévia (Pestana e Vieira, 2018), onde foi investigado a aceitação da distribuição de tarefas crowdsourcing baseado no modelo (ConTask Model). No modelo previamente desenvolvido foram tratados aspectos referentes as principais entidades crowdsourcing e 5 dimensões contextuais, segundo Zimmermann et al. (2007) que podem afetar a distribuição de tarefas: Localização, Tempo, Individualidade, Atividade e Relação.

A dimensão Atividade refere-se às atividades atuais e futuras nas quais as entidades estão envolvidas. A dimensão atividade sugere que as tarefas podem ser distribuídas de acordo com a atividade atual do usuário, como enviar tarefas para o grupo que estão viajando de ônibus ou para aqueles que estão usando um projetor, em um determinado momento.

A dimensão Relação captura as relações que uma entidade estabeleceu para outras entidades, o que torna possível definir uma relação entre duas entidades como critérios para a distribuição de tarefas. A reputação do trabalhador no crowdsourcing é um tópico muito frequente estudado na literatura (Mrazovic e Matskin, 2015; Lee et al., 2015). Por exemplo, nas redes sociais, a relação pode ser profissional como um professor, ou pessoal, tal como amizade.

A dimensão de Tempo pode permitir disparar tarefas em intervalos de tempo, mas também configurar períodos específicos do ano, mês ou semana. Esses elementos podem ser usados pelo solicitante como critérios para que uma tarefa seja entregue ou não, como quando a tarefa é exclusiva para ser feita à noite, em uma determinada hora ou no final de semana.

A dimensão de Local associa uma tarefa a uma condição espacial para permitir a distribuição apenas para os trabalhadores ou dispositivos localizados nesses locais. Por exemplo, os trabalhadores podem receber tarefas relacionadas ao espaço em que estão atualmente inseridas, como "na universidade" ou "em um local ensolarado". De acordo com Krumm (2010), a localização absoluta refere-se à tarefa a ser recebida em uma área geográfica limitada por coordenada geográfica, enquanto a localização representativa pode ser especificada pelas propriedades do local, como endereços, nomes, etc.

A dimensão Individualidade compreende tudo o que pode ser observado sobre uma entidade, tipicamente seu estado. Esse tipo de informação de contexto pode ser usado na distribuição de tarefas quando a tarefa requer usuários com perfis específicos (idade, língua, especialização, nível profissional, estado civil, nascimento, idioma, experiência, nacionalidade, gênero ou ocupação) ou dispositivos com um determinado tipo de configuração. Por exemplo, trabalhadores idosos ou dispositivos que contenham um $3 \mathrm{G}$ ativo e uma câmera de 10 megapixels.

Uma avaliação foi feita para identificar a aceitação do usuário de acordo com alguns parâmetros definidos no modelo. A localização do contribuinte foi tratada de acordo com a distância entre o participante e o local tratado na tarefa; Também foi avaliado a aceitação quanto a frequência do contribuinte no local tratado na tarefa; Outro aspecto tratado foi o relacionamento entre o requerente da tarefa e o contribuinte, de acordo com a conexão social entre eles; Atividade atual executada no momento do recebimento da 
tarefa; O turno em que foi recebida a tarefa também foi tratado no modelo; por fim, também foi tratado o nível de expertise do contribuinte.

O modelo de Guo (2018) reúne uma investigação secundária com trabalhos relacionados a alocação de tarefas crowdsourcing. Primeiramente ele define um modelo conceitual (2018), e depois disserta sobre os desafios da área. O modelo é dividido baseado em três principais entidades: tarefas, trabalhador e servidor.

O modelo de tarefas é caracterizado pelo Tipo da tarefa (urgente/normal, pontual/regional, estática/dinâmica), pelo domínio (tradução, organização, ...) e preço (escalabilidade, dificuldade, ...), pelos contextos espaço-temporal (posição pontual, posição regional, tempo de início, tempo de fim) e pelos métodos de execução das tarefas (individual, compartilhamento, comunidade, ...).

O modelo do trabalhador pode ser caracterizado pelos contextos espaço temporal (posição atual, histórico da trajetória, predição da trajetória, pontos de interesse, ...), pela informação temporal (tempo de trabalho, tempo livre, ...), confiabilidade (dados do histórico, tipo da tarefa e indicador do valor da tarefa), habilidades (variadas habilidades, nível de habilidade, ...), Preferencia (tipo da tarefa, localização, tempo).

Quanto ao modelo do servidor, este pode ser caracterizado pelo armazenamento de dados e pela alocação de tarefas. No armazenamento de dados, guarda-se as informações da tarefa providas pelo solicitante e informações do trabalhador coletadas e processadas via mineração de dados. No processo de alocação de tarefas há modelos de otimização que visam maximizar a qualidade das tarefas que são concluídas (a partir do número de participantes e da cobertura das tarefas, por exemplo), minimizando o custo do sistema (com dispositivos, com trabalhadores, ...), restringindo a alocação de acordo com o tempo (tempo inicial e final das tarefas, tempo livre do trabalhador, ...), posicionamento (posição atual, distância a percorrer, ...).

Os desafios destacados na pesquisa foram: alocação de tarefas individual ou múltipla de acordo com critérios pré-definidos; alocação de tarefas com baixo custo para reduzir o consumo de energia requerido por esses sistemas; alocação de tarefas de forma a aprimorar a qualidade dos resultados obtidos a partir de algoritmos de alocação ou mecanismos de incentivo.

O modelo apresentado por Guo (2018) não chegou a ser avaliado. O trabalho, porém, contemplou uma vasta compilação de trabalhos que fizeram avaliações de alocação de tarefas, tanto através de experimentações em plataformas ou aplicações crowdsourcing existentes, quanto através de grandes bancos de dados sintéticos.

\section{Metodologia}

O método utilizado para realização do experimento e avaliação dos resultados será baseado no User Experience (Roto et al, 2009), com algumas possíveis alterações para o domínio geral: crowdsourcing. Para comprovar a generalidade dos modelos, o experimento será executado de acordo com os problemas aplicados a três diferentes domínios: Cidades Inteligentes, Saúde e Educação, que são áreas onde se vê a colaboração da sociedade para resolver problemas. Para que haja alta aderência de contribuintes no experimento, serão escolhidas aplicações/plataformas crowdsourcing 
que sejam já amplamente estabelecidas nesses domínios, e possam servir para atingir o objetivo desta experimentação.

A Figura 2 descreve como se dará a avaliação. Nessa avaliação, será registrado aspectos da experiência do usuário (contribuinte crowdsourcing e solicitante) ao utilizar essas aplicações, e investigar como as aceitações serão afetadas a partir de variações das características referentes a tarefa e o contribuinte. Essas variações são relacionadas a aspectos contextuais descritas no modelo contextual previamente desenvolvido, aprimorado pelo que foi destacado em Guo (2018). Por fim, verificaremos aquelas situações em que houve aceitação, em detrimento daquelas que não ocorreram, de acordo com cada domínio proposto e com cada tarefa analisada nos experimentos.

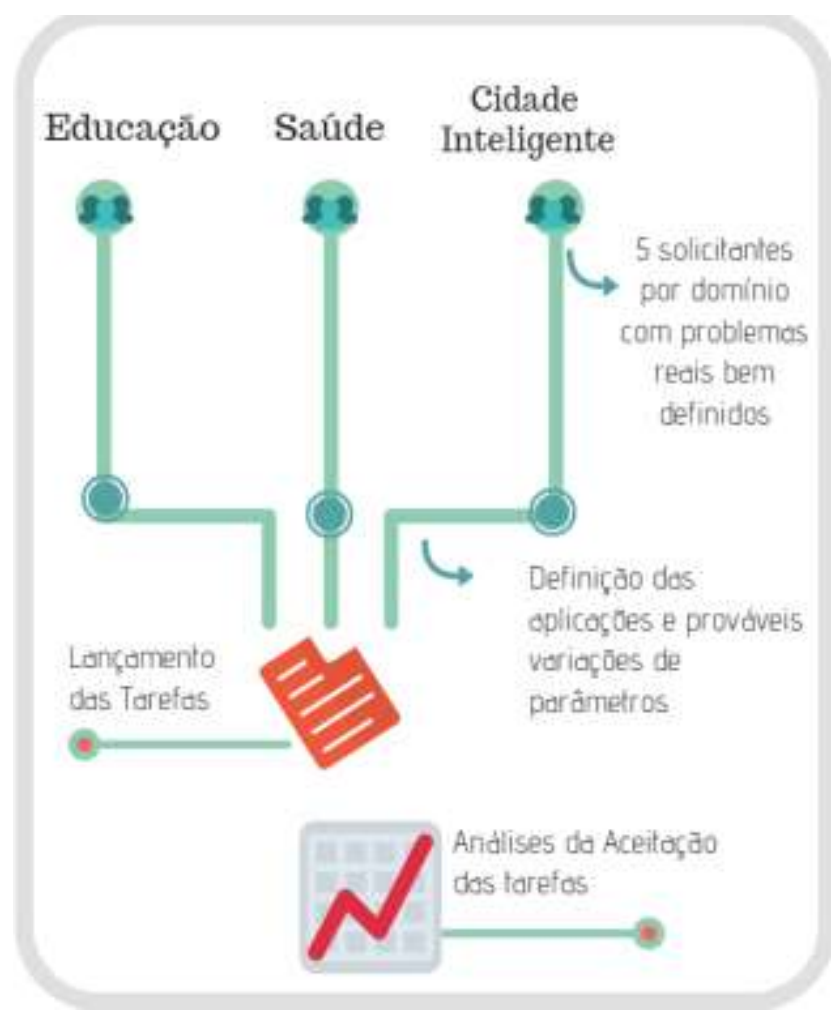

Figura 3. Modelo conceitual descrito em Guo apresentando suas três principais entidades (Task, Worker and Server) e caracterizando cada uma delas

A estratégia User Experience (Roto et al, 2009) utiliza-se de vários métodos diferentes para coletar dados ricos dos usuários. Por exemplo, é possível combinar dados de observação com os dados de registro do sistema ou com dados de insights subjetivos de entrevistas ou questionários. A combinação de observações do usuário seguida de entrevistas é bastante utilizada como método de UX. As técnicas de UX vão além das observações quanto as ações do usuário (usabilidade), buscando um alcance associado a como o usuário se sente, observando suas atividades físicas, sua interação social e foco de atenção, depois entrevistando e registrando os pareceres combinados com dados objetivos.

Segundo Roto el al. (2009), nos experimentos acadêmicos, é necessário que haja rigor científico, portanto, um requisito central para o UX é ter um método de avaliação. Exemplos de métodos academicamente válidos foram estudo piloto de longo prazo, experiência de amostragem desencadeada por eventos e instrumento de avaliação. Além 
disso, os métodos devem preferencialmente permitir estudos repetíveis e comparativos em uma maneira iterativa com ciclos de desenvolvimento de produto, no design de pesquisa que precisa de ferramentas de avaliação eficazes para iterações.

\section{Resultados Esperados}

Através da aplicação do experimento, poderá ser possível elencar algum dos parâmetros utilizados nos sistemas crowdsourcing que sejam decisivos para a aceitação das tarefas. Esses parâmetros podem ser organizados em forma de modelo onde seja possível visualizar aqueles que tiveram maior destaque na aceitação das tarefas e diferenciar daqueles que tiveram menor destaque.

Através desse estudo, vamos implementar tais considerações como aprimoramento da aplicação ConTask App, para que esta possa ser estabelecida como uma plataforma de distribuição de tarefas crowdsourcing, em parceria com uma ou mais instituições que necessite desses serviços. Essa implementação abriria novos caminhos para avaliar o desempenho do ConTask App como plataforma crowdsourcing baseando-se na estratégia UX.

\section{Referências}

Brabham, D. (2013) Using Crowdsourcing In Government, IBM Cent. Bus. Gov., p. 142.

Guo, B., Liu, Y., Wang L., Li, V. O. K., Lam, J. C. K. e Yu, Z. (2018) "Task Allocation in Spatial Crowdsourcing: Current State and Future Directions", em: IEEE Internet of Things Journal, vol. 5, no. 3, pp. 1749-1764

Howe, J. (2006) The Rise of Crowdsourcing, Wired Mag., v. 14, n. 06, p. 1-5.

Krumm, J. (2010) Ubiquitous Computing Fundamentals, 285 p. ISBN

9781420093605.

Lee, C., Chan, C., Ho, S., Choy, K., Ip, W. (2015) "Explore the feasibility of adopting crowdsourcing for innovative problem solving", Industrial Management \& Data Systems, v. 115,n. 5, p. 803-832. https://doi.org/10.1108/IMDS-09-2014-0249i.

Pestana M. C. e Vieira V. (2018) "Context-Aware Task Distribution for Mobile Crowdsourcing", em: Proceedings of the 17th Brazilian Symposium on Human Factors in Computing Systems (IHC 2018). ACM, New York, NY, USA, Article 14, 8 pages. DOI: https://doi.org/10.1145/3274192.3274206

Mrazovic, P. e Matskin, M. (2015) "MobiCS: Mobile Platform for Combining Crowdsourcing and Participatory Sensing", em: IEEE 39th Annual Computer Software and Applications Conference. IEEE, 553-562. https://doi.org/10.1109/COMPSAC.2015.2

Roto, V., Obrist, M. e Väänãnen-Vainio-Mattile, K. (2009) "User. Experience Evaluation Methods", Academic and Industrial Contexts, em: Proceedings of Workshop UXEM'09, CHI'09, Boston, USA

Zimmermann, A. L., Oppermann R., and Sankt A. (2007) “An Operational Definition of Context. Context”, 558-571. 